\title{
Balloon Valvuloplasty for Bioprosthetic Tricuspid Valve Stenosis
}

\author{
Emmanouil Petrou, MD, Vasiliki Vartela, MD, Chrysafios Girasis, MD, \\ Maria Boutsikou, MD, loannis lakovou, MD, and Gregory Pavlides, MD \\ Cardiology Department, Onassis Cardiac Surgery Center, Athens, Greece
}

A 73-year-old woman was admitted to our hospital for a scheduled percutaneous transcatheter balloon valvuloplasty of a stenotic tricuspid bioprosthesis. The patient had a history consistent with exertional dyspnea, abdominal distension, and edema of the lower extremities resistant to diuretic treatment over the previous few months. Forty-three years prior, she underwent aortic valve replacement with a Starr-Edwards mechanical prosthetic valve, with a mean pressure gradient of $25 \mathrm{~mm} \mathrm{Hg}$, for aortic stenosis of rheumatic etiology (Fig. 1, white arrow). Furthermore, three years after aortic valve replacement the patient underwent mitral valve replacement with a bileaflet mechanical valve for mitral stenosis which had a stenotic portion (mean pressure gradient $10 \mathrm{~mm} \mathrm{Hg}$ ) (Fig. 1, black arrow), and tricuspid valve replacement with a CarpentierEdwards bioprosthesis for tricuspid stenosis (Fig. 1, yellow arrow).
On admission, the patient was on atrial fibrillation and physical examination was compatible with right heart failure. Echocardiography demonstrated severe tricuspid stenosis and a mild regurgitation. The mean diastolic gradient across the tricuspid valve was $15.4 \mathrm{~mm}$ $\mathrm{Hg}$ (Fig. 2A and B). Therefore, we proceeded to percutaneous valvuloplasty of the tricuspid valve using fluoroscopy, under regional anesthesia and sedation, inflating a $15 \times 40-\mathrm{mm}$ Inoue balloon up to a pressure of $15 \mathrm{~atm}$ (Fig. 1B). ${ }^{1{ }^{12)}}$ According to hemodynamic measurements, the gradient across the bioprosthetic tricuspid valve dropped from $13.0 \mathrm{~mm} \mathrm{Hg}$ before to $6.0 \mathrm{~mm} \mathrm{Hg}$ after dilation (Fig. $3)$, and on repeat echocardiography the mean diastolic gradient was decreased to $7.2 \mathrm{~mm} \mathrm{Hg}$ without worsening of the regurgitation (Fig. 2C and D). The patient's symptoms soon improved; however, two months later she experienced a massive pulmonary hemorrhage
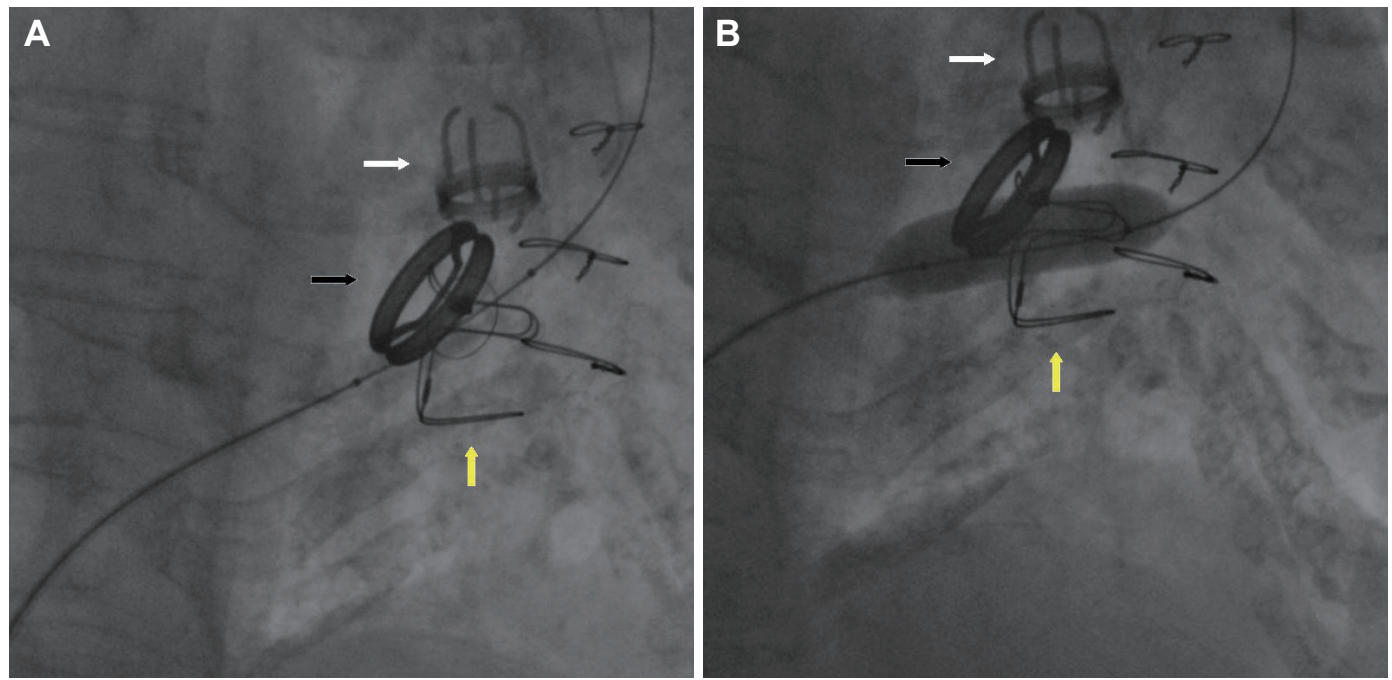

Fig. 1.

Received: July 29, 2013 / Revision Received: September 3, 2013 / Accepted: October 8, 2013

Correspondence: Emmanouil Petrou, MD, Cardiology Department, Onassis Cardiac Surgery Center, 356 Sygrou Ave., GR-17674, Kallithea-Athens, Greece Tel: 306945112509, Fax: 302102751028,E-mail: emmgpetrou@hotmail.com

- The authors have no financial conflicts of interest.

This is an Open Access article distributed under the terms of the Creative Commons Attribution Non-Commercial License (http://creativecommons.org/licenses/ by-nc/3.0) which permits unrestricted non-commercial use, distribution, and reproduction in any medium, provided the original work is properly cited. 


\section{C Korean Circulation Journal}

while on heparin and acenocoumarol within therapeutic range, and died after a long hospitalization in the intensive care unit.

\section{References}

1. Egred $M$, Albouaini $K$, Morrison WL. Images in cardiovascular medicine.
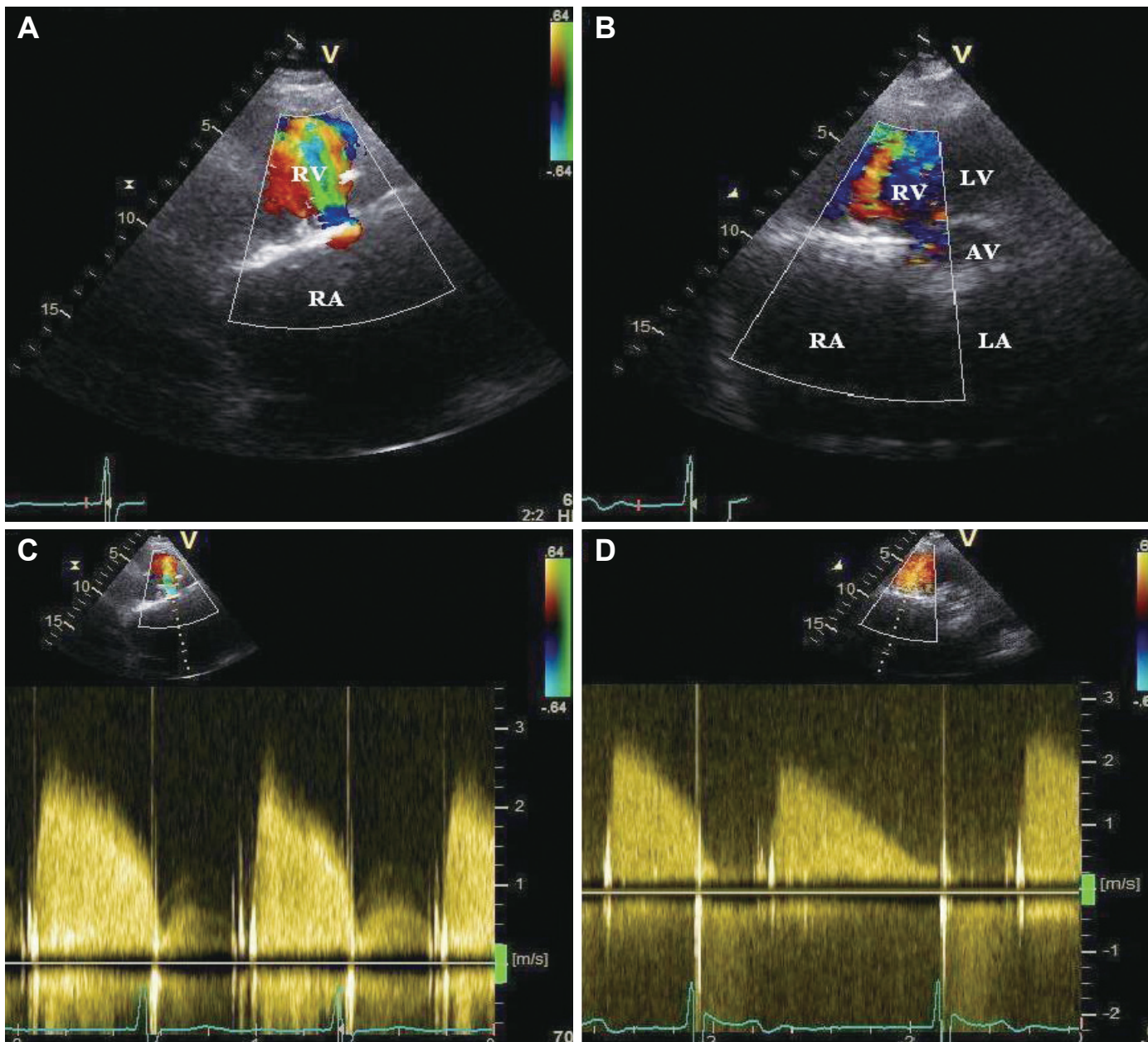

Fig. 2.
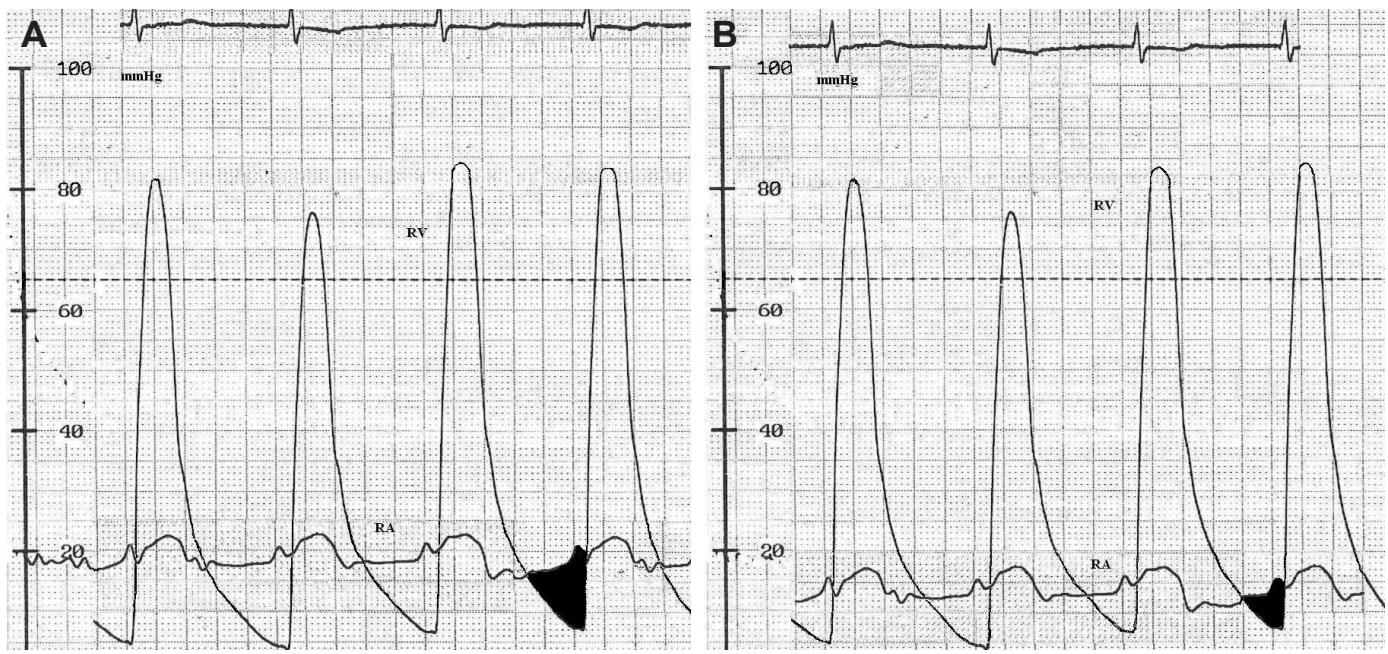

Fig. 3.
Balloon valvuloplasty of a stenosed bioprosthetic tricuspid valve. Circulation 2006;113:e745-7.

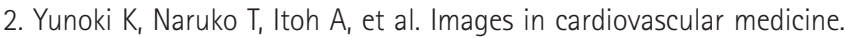
Percutaneous transcatheter balloon valvuloplasty for bioprosthetic tricuspid valve stenosis. Circulation 2006;114:e558-9. 